Provided for non-commercial research and education use. Not for reproduction, distribution or commercial use.

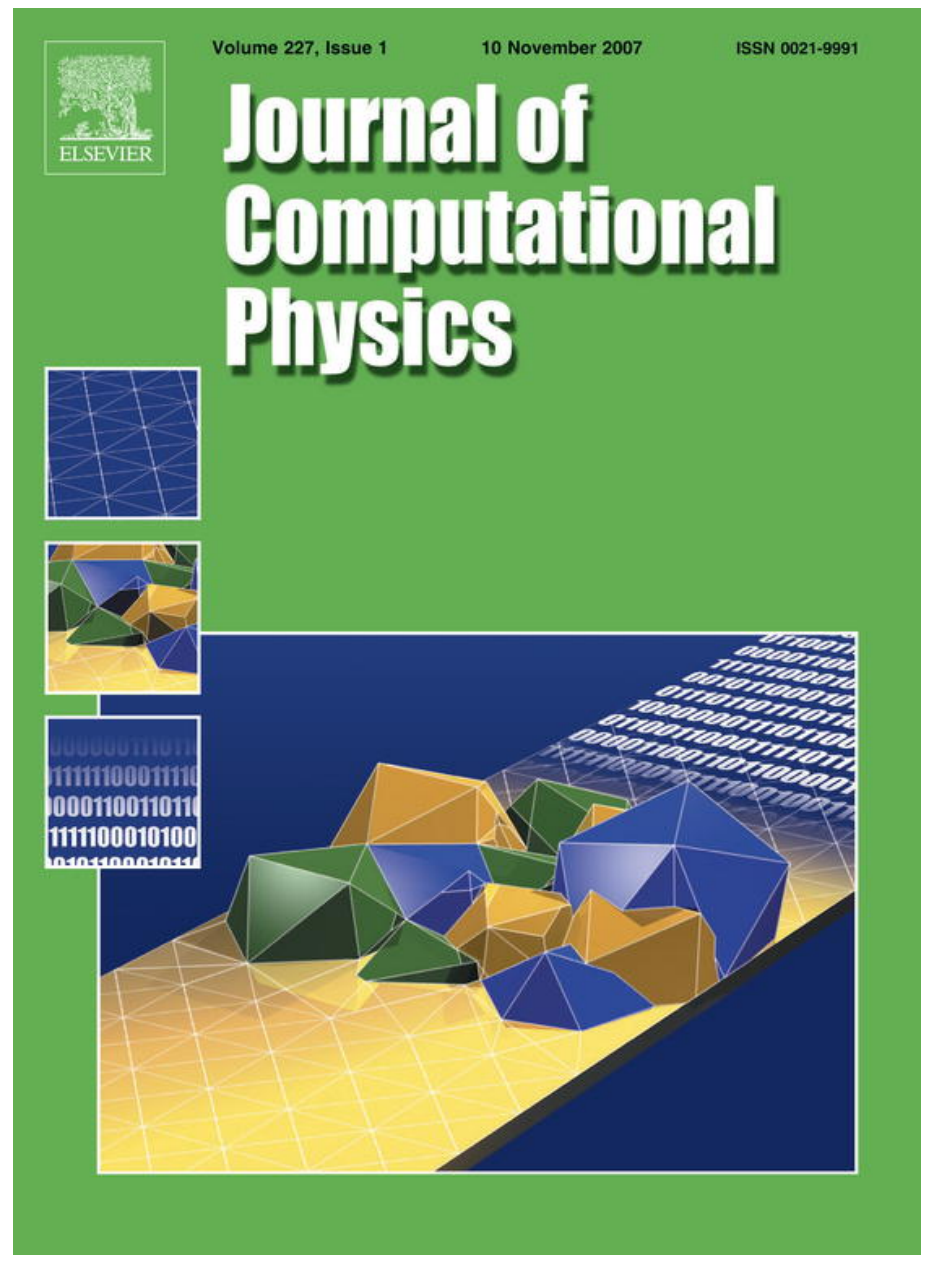

This article was published in an Elsevier journal. The attached copy

is furnished to the author for non-commercial research and education use, including for instruction at the author's institution, sharing with colleagues and providing to institution administration.

Other uses, including reproduction and distribution, or selling or licensing copies, or posting to personal, institutional or third party websites are prohibited.

In most cases authors are permitted to post their version of the article (e.g. in Word or Tex form) to their personal website or institutional repository. Authors requiring further information regarding Elsevier's archiving and manuscript policies are encouraged to visit: 


\title{
A faster aggregation for 3D fast evanescent wave solvers using rotations
}

\author{
Ignace Bogaert *, Davy Pissoort, Femke Olyslager \\ Department of Information Technology (INTEC), Ghent University, Sint-Pietersnieuwstraat 41, B-9000 Ghent, Belgium
}

Received 28 November 2006; received in revised form 6 June 2007; accepted 1 August 2007

Available online 14 August 2007

\begin{abstract}
A novel technique to accelerate the aggregation and disaggregation stages in evanescent plane wave methods is presented. The new method calculates the six plane wave radiation patterns from a multipole expansion (aggregation) and calculates the multipole expansion of an incoming field from the six plane wave incoming field patterns. It is faster than the direct approach for multipole orders larger than one, and becomes six times faster for large multipole orders. The method relies on a connection between the discretizations of the six integral representations, and on the fact that the Wigner D-matrices become diagonal for rotations around the $z$-axis. The proposed technique can also be extended to the vectorial case in two different ways, one of which is very similar to the scalar case. The other method relies on a Beltrami decomposition of the fields and is faster than the direct approach for any multipole order. This decomposition is also not limited to evanescent wave solvers, but can be used in any vectorial multilevel fast multipole algorithm.
\end{abstract}

(c) 2007 Elsevier Inc. All rights reserved.

Keywords: Stable plane wave method; Spectral representation of the Green function; Evanescent waves; Multipoles; Rotation; (Dis)aggregation

\section{Introduction}

Currently, several so-called Multilevel Fast Multipole Algorithms (MLFMAs) exist [1-7]. These methods accelerate the matrix-vector multiplications required for the iterative solution of the linear systems of equations arising in Method of Moments based integral-equation solvers. Unfortunately, most of these methods only apply to a specific type of problem. Multilevel fast multipole methods based on homogeneous plane wave expansions of the Green function (the so-called plane wave method) reduce the computation time and memory requirements for high frequency (HF) problems to $\mathcal{O}(N)-N$ denotes the number of unknowns - or $\mathcal{O}(N \log N)$, but completely fail for low frequency (LF) problems due to roundoff errors. On the other hand, Multilevel Fast Multipole Algorithms based on multipole expansions of the Green function reduce both computation time and memory requirements to $\mathcal{O}(N)$ for LF problems, but not for HF problems. Hence, they are referred to as the low

\footnotetext{
* Corresponding author. Tel.: +329 2643354; fax: +32 92649969.

E-mail address: Ignace.Bogaert@intec.ugent.be (I. Bogaert).
} 
frequency MLFMAs (LF-MLFMAs). To mitigate this a new class of MLFMAs was introduced, which all somehow rely on a discretized version of the spectral representation of the Green function. These methods, most notably the stable plane wave method [6,8], are capable of delivering $\mathcal{O}(N)$ or $\mathcal{O}(N \log N)$ complexity for LF and HF problems, respectively. However, as shown in [9], the plane wave method turns out to be the most efficient MLFMA if the frequency is high enough to reach the target accuracy. Therefore, the state of the art is to introduce a level in the MLFMA tree above (the HF part) which the plane wave method is used, and below (the LF part) which either the LF-MLFMA (as for example in [10]) or some form of evanescent plane wave method (as for example in [9]) is used. In [8,9], it is argued that the evanescent wave technique is more efficient than the LFMLFMA, especially for high accuracies. The reason for this is the diagonality of the translation operator, which reduces the cost of one translation from $\mathcal{O}\left(L^{4}\right)$ to $\mathcal{O}\left(L^{2}\right)$ with $L$ the multipole order (of the LF-MLFMA). This shows that evanescent wave methods certainly are of practical importance for the LF part of MLFMAs.

However, their main drawback is that the underlying Green function's integral representation only converges in one half-space of choice. By consequence, in practice, six integral representations are needed to cover the entire space. For the propagating plane waves, all dependency on the half-space can be incorporated into the translation operator, thereby allowing to calculate all six integral representations starting from only one radiation pattern. Hence, for the propagating plane waves, only one radiation pattern is needed. For the evanescent plane waves up to now no such technique exists despite several attempts to solve this conundrum. For example, in [11], Jiang and Chew split the evanescent plane waves into so called 'shallow evanescent plane waves' and 'deep evanescent plane waves'. The shallow plane waves are obtained by extrapolating the propagating plane waves and improve the efficiency of the method at higher levels. However for very low frequencies or for very low levels in the tree, the extrapolation has little use, since virtually none of the evanescent plane waves will be shallow. Indeed, if the extrapolation would still be effective at very low frequencies, the plane wave method would to some extent still work at those frequencies. Also, according to [11], it is not possible to calculate all six integral representations of the deep evanescent plane waves starting from only one radiation pattern. The need for six radiation patterns results in high memory requirements [10] and a high computational cost. As is done in $[9,12]$, the memory problem can be reduced by first calculating a multipole expansion of the radiated field, and then going to the plane wave basis. The calculation of the six evanescent radiation patterns from a multipole expansion will from here on be referred to as 'aggregation'. The reverse procedure, namely projecting the six evanescent incoming field patterns of an MLFMA group onto multipoles, will be referred to as 'disaggregation'. These procedures not only arise in the LF-part of [9], but can also be used when for example multiple scattering from a collection of spheres is considered (where the sources in every MLFMA group are multipoles). It is thus important to do the (dis)aggregation as efficiently as possible.

The main goal of this paper is to introduce an elegant, novel and exact method to do this (dis)aggregation. This new method relies on the use of Wigner rotation matrices and the fact that these matrices are diagonal for rotations around the $z$-axis. The computation time is lower than the direct computation if the maximal multipole order $L$ is larger than one, which is true for all practical cases. Moreover, if a large enough number of multipoles are used, the computation time is reduced by a factor six compared to the direct case. It will also be shown that the proposed scheme applies to both the scalar (for example acoustics) and the vectorial (for example electromagnetics) case. In the latter case, two methods are provided, the second of which is faster than the direct approach for any multipole order. The proposed methods do not reduce the complexity however, so they can only be used for the LF-part of the MLFMA tree. A drawback of the method occurs when one is simulating a structure which is long in one direction. Only a few of the six radiation patterns are needed then, while the proposed method still calculates all six radiation patterns. Such inefficiencies can be avoided by switching to the direct method for these cases.

Notation: throughout this paper all sources and fields are assumed time-harmonic with angular frequency $\omega$; temporal dependencies $\mathrm{e}^{\mathrm{j} \omega t}$ are suppressed. Unit vectors are denoted by $\boldsymbol{u}_{\tau}$, with $\tau=x, y, z$.

\section{MLFMAs with evanescent plane waves}

MLFMAs with evanescent plane waves [6,8,11], like all MLFMA codes, uses a hierarchical cubical subdivision of the computational domain. For the interaction between groups on a level, it relies upon the spectral decomposition of the spherical Hankel function of the second kind and of zeroth order: 


$$
h_{0}^{(2)}(\gamma r)=\frac{1}{2 \pi} \int_{-\infty}^{\infty} \int_{-\infty}^{\infty} \mathrm{e}^{\mp \mathrm{j} k \cdot r} \frac{\mathrm{d} k_{x} \mathrm{~d} k_{y}}{\gamma k_{z}(\gamma, K)}, \quad z=\boldsymbol{u}_{z} \cdot \boldsymbol{r} \gtrless 0 .
$$

Here, $\gamma$ is the free-space wavenumber, $\boldsymbol{k}=\left(k_{x}, k_{y}, k_{z}\right)$ the wave vector, and $\boldsymbol{r}$ a global position vector. However, $k_{z}$ is a quantity depending on $\gamma$ and $K=\sqrt{k_{x}^{2}+k_{y}^{2}}$, defined as

$$
k_{z}(\gamma, K)= \begin{cases}\sqrt{\gamma^{2}-K^{2}}, & \gamma \geqslant K \\ -\mathrm{j} \sqrt{K^{2}-\gamma^{2}}, & K>\gamma\end{cases}
$$

In the stable plane wave method, integral (1) is split in a propagating and an evanescent part by means of polar coordinates $\left(k_{x}, k_{y}\right)=(K \cos (\phi), K \sin (\phi))$ :

$$
\begin{aligned}
h_{0}^{(2)}(\gamma r) & =\frac{1}{2 \pi} \int_{0}^{2 \pi} \int_{0}^{\infty} \mathrm{e}^{\mathrm{f} \mathrm{j} k \cdot r} \frac{K \mathrm{~d} K \mathrm{~d} \phi}{\gamma k_{z}(\gamma, K)} \\
& =\underbrace{\frac{1}{2 \pi} \int_{0}^{2 \pi} \int_{0}^{\gamma} \mathrm{e}^{\mathrm{fj} \boldsymbol{k} \cdot \boldsymbol{r}} \frac{K \mathrm{~d} K \mathrm{~d} \phi}{\gamma \sqrt{\gamma^{2}-K^{2}}}}_{\text {Propagating }}+\underbrace{\frac{\mathrm{j}}{2 \pi} \int_{0}^{2 \pi} \int_{\gamma}^{\infty} \mathrm{e}^{\mp \mathrm{j} k \cdot r} \frac{K \mathrm{~d} K \mathrm{~d} \phi}{\gamma \sqrt{K^{2}-\gamma^{2}}}}_{\text {Evanescent }} .
\end{aligned}
$$

Then both integrals are discretized separately, for example for the case $z>0$ :

$$
h_{0}^{(2)}(\gamma r)=\sum_{v_{p}=1}^{V_{p}} w_{v_{p}}^{p} \mathrm{e}^{-\mathrm{j} \boldsymbol{k}_{v_{p}}^{p} \cdot \boldsymbol{r}}+\sum_{v_{e}=1}^{V_{e}} w_{v_{e}}^{e} \mathrm{e}^{-\mathrm{j} \boldsymbol{k}_{v_{e}}^{e} \cdot \boldsymbol{r}}
$$

where $w_{v_{p}}^{p}, \boldsymbol{k}_{v_{p}}^{p}, w_{v_{e}}^{e}$ and $\boldsymbol{k}_{v_{e}}^{e}$ are the weights and nodes of the propagating and evanescent part, respectively. The discretization of the propagating part is straightforward [6]. The discretization of the evanescent part is more difficult, since the evanescent integral in (4) covers an infinite region. However, since $k_{z}(\gamma, K)=-\mathrm{j} \sqrt{K^{2}-\gamma^{2}}$, the integral converges in an exponential manner if $z \gtrless 0$. This allows the discretization to be done with exponential accuracy. It can be done in many ways, for example with a singular value decomposition (SVD) based approach or uniform discretization, both described in [6]. However, the details of the discretization are not important for the remainder of this paper.

An important property of (1) is that it is valid only if $z \gtrless 0$. In the plane $z=0$, this integral representation does not converge. For the discretized version, matters are even worse, since series (5) will only converge if $\boldsymbol{r}$ remains far enough from the plane $z=0$. A more rigorous criterium for convergence of (5) is given in [6], with some positive constant $C$ :

$$
\sqrt{x^{2}+y^{2}}<C z
$$

As a consequence, only a cone-like region around the $z$-axis can be covered with this expansion of the Green function. This situation is remedied by introducing extra integral (series) representations which converge along the $x$ - and $y$-axes. As a result, in total six integral representations are used, which will henceforth be denoted as $1 \pm, 2 \pm$, and $3 \pm$. This configuration has been ubiquitously used in the literature $[6,8,11]$. In [6], it is shown that in an oct-tree using these six integral representations, criterium (6) is always satisfied with $C=\sqrt{2}$, thus assuring convergence of the series. Fig. 1 shows an example configuration in which the field in the point $\boldsymbol{d}_{1}$ due to sources $\boldsymbol{J}$ in the box must be calculated by means of the $3+$ expansion, while the fields in the point $\boldsymbol{d}_{2}$ must be calculated by means of the $1+$ expansion.

The propagating parts of the six series are all integrations over one half of the Ewald sphere. As is shown in [6] this permits absorbing all directional dependence into the weights. As a consequence only one radiation pattern is needed for the propagating part. Unfortunately, this is not possible for the evanescent part [11]. Therefore, from now on, we will focus on the evanescent part only, so $w_{v_{e}}^{e}$ and $\boldsymbol{k}_{v_{e}}^{e}$ will henceforth be written as $w_{v}$ and $\boldsymbol{k}_{v}$. In this paper, it will be shown that the six evanescent radiation patterns can be calculated from a multipole expansion with asymptotically the same computational cost as for one radiation pattern, thus gaining a factor six. The essential element of this novel method is the fact that the nodes of the different representations are rotated versions of the nodes for the expansion around the positive $z$-axis $\boldsymbol{k}^{v}\left(=\boldsymbol{k}_{v}^{3+}\right)$ : 


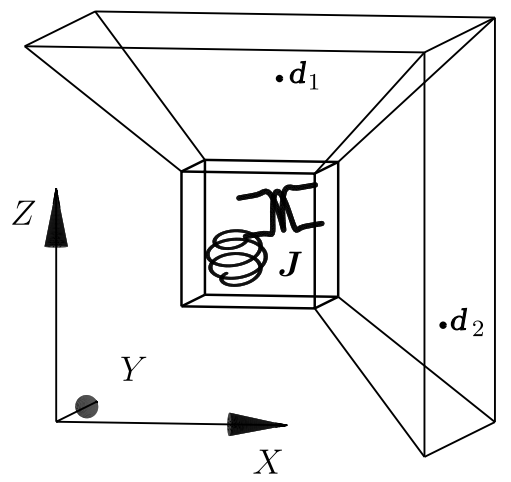

Fig. 1. The radiated fields of the central box is given by different expansions in the different cone-like regions. For example, the fields in $\boldsymbol{d}_{1}$ must be calculated using the $3+$ expansion while the fields in $\boldsymbol{d}_{2}$ must be calculated by means of the $1+$ expansion.

$$
\begin{aligned}
\boldsymbol{k}_{v}^{1+} & =\mathbf{R}_{x}^{-1} \cdot \boldsymbol{k}_{v}, \\
\boldsymbol{k}_{v}^{2+} & =\mathbf{R}_{y}^{-1} \cdot \boldsymbol{k}_{v}, \\
\boldsymbol{k}_{v}^{3+} & =\mathbf{R}_{z}^{-1} \cdot \boldsymbol{k}_{v}=\boldsymbol{k}_{v},
\end{aligned}
$$

In addition:

$$
\boldsymbol{k}_{v}^{p-}=-\boldsymbol{k}_{v}^{p+} \quad \forall p \in\{1,2,3\} .
$$

with the matrices $\mathbf{R}_{x}, \mathbf{R}_{y}$ and $\mathbf{R}_{z}$ defined as

$$
\begin{aligned}
& \mathbf{R}_{x}=\mathbf{R}\left(-\frac{2 \pi}{3}, \frac{1}{\sqrt{3}}\left(\boldsymbol{u}_{x}+\boldsymbol{u}_{y}+\boldsymbol{u}_{z}\right)\right)=\left[\begin{array}{lll}
0 & 1 & 0 \\
0 & 0 & 1 \\
1 & 0 & 0
\end{array}\right], \\
& \mathbf{R}_{y}=\mathbf{R}\left(\frac{2 \pi}{3}, \frac{1}{\sqrt{3}}\left(\boldsymbol{u}_{x}+\boldsymbol{u}_{y}+\boldsymbol{u}_{z}\right)\right)=\left[\begin{array}{lll}
0 & 0 & 1 \\
1 & 0 & 0 \\
0 & 1 & 0
\end{array}\right] . \\
& \mathbf{R}_{z}=\mathbb{1}
\end{aligned}
$$

In this, the rotation matrix $\mathbf{R}(\alpha, \boldsymbol{u})$ is a $3 \times 3$ matrix which rotates a vector $\boldsymbol{x}$ around the axis $\boldsymbol{u}$ (with $\boldsymbol{u} \cdot \boldsymbol{u}=1$ ) over the angle $\alpha$ into the vector $\boldsymbol{x}^{\prime}$, viz. $\boldsymbol{x}^{\prime}=\mathbf{R}(\alpha, \boldsymbol{u}) \cdot \boldsymbol{x}$. The direction of rotation is determined by means of the right hand rule. A graphical representation of this is given in Fig. 2. It will now be shown that the relationships (7)-(10) can be unified in one symmetric expression. Begin by observing that $\mathbf{R}_{x}, \mathbf{R}_{y}$, and $\mathbf{R}_{z}$ are all rotations around the $\frac{1}{\sqrt{3}}\left(\boldsymbol{u}_{x}+\boldsymbol{u}_{y}+\boldsymbol{u}_{z}\right)$-axis. This means they all can be converted into rotations around the $z$-axis by means of one similarity transform. With $\mathbf{R}_{0}=\mathbf{R}\left(-\arccos \left(\frac{1}{\sqrt{3}}\right), \frac{1}{\sqrt{2}}\left(-\boldsymbol{u}_{x}+\boldsymbol{u}_{y}\right)\right)$, this yields

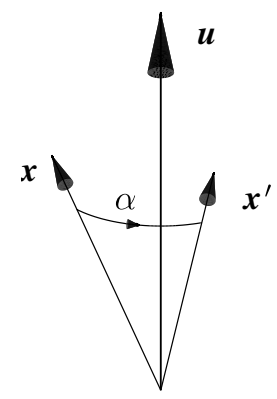

Fig. 2. The rotation matrix $\mathbf{R}(\alpha, \boldsymbol{u})$ rotates a vector $\boldsymbol{x}$ around the axis $\boldsymbol{u}$ over the angle $\alpha$ into the vector $\boldsymbol{x}^{\prime}$. The direction of the rotation is determined by the right hand rule. 


$$
\begin{aligned}
& \mathbf{R}_{x}=\mathbf{R}_{0}^{-1} \cdot \mathbf{R}\left(-\frac{2 \pi}{3}, \boldsymbol{u}_{z}\right) \cdot \mathbf{R}_{0}, \\
& \mathbf{R}_{y}=\mathbf{R}_{0}^{-1} \cdot \mathbf{R}\left(-\frac{4 \pi}{3}, \boldsymbol{u}_{z}\right) \cdot \mathbf{R}_{0}, \\
& \mathbf{R}_{z}=\mathbf{R}_{0}^{-1} \cdot \mathbf{R}\left(-\frac{6 \pi}{3}, \boldsymbol{u}_{z}\right) \cdot \mathbf{R}_{0} .
\end{aligned}
$$

This means that the discretization points for all six representations can be unified in one formula:

$$
\boldsymbol{k}_{v}^{p s}=\mathbf{R}_{0}^{-1} \cdot\left[s \mathbf{R}\left(\frac{2 p \pi}{3}, \boldsymbol{u}_{z}\right)\right] \cdot \mathbf{R}_{0} \cdot \boldsymbol{k}_{v}^{3+} \quad \forall s \in\{-1,+1\} \quad \forall p \in\{1,2,3\} .
$$

When $s$ is used as an index, for example in $\boldsymbol{k}_{v}^{p s}$, the +1 and -1 are denoted as + and - , respectively. Formula (17) is a form in which the symmetry between the six radiation patterns is visible. This symmetry will be crucial in developing the accelerated scheme, since it will allow the six radiation patterns to be decomposed in six other patterns which require only one sixth of the amount of work to calculate. The derivations will be presented in the following sections.

\section{A faster (dis)aggregation for the scalar case}

As stated in the introduction, the aggregation denotes the calculation of the six evanescent radiation patterns from a multipole expansion. This process is used and elaborated in [9]. It consists of calculating

$$
\Theta_{v}^{p s}=\sum_{l=0}^{L} \sum_{m=-l}^{l} a_{l m} j^{l} Y_{l, m}\left(\boldsymbol{k}_{v}^{p s}\right) \quad \forall p \in\{1,2,3\}, \quad s \in\{-1,+1\},
$$

where the $a_{l m}$ are the multipole coefficients, $Y_{l, m}(\boldsymbol{k})$ denotes a spherical harmonic and $L$ is the maximum multipole order that is taken into account. The $\boldsymbol{k}_{v}^{p s}$ are the discretization nodes from the evanescent part in (5). The definition and some properties of the spherical harmonics are summarized in the Appendix. When $\Phi_{v}^{p s}$ denotes the incoming field patterns in the six directions, the disaggregation is given by

$$
\begin{aligned}
b_{l m} & =\sum_{v} w_{v} \sum_{p, s} \Phi_{v}^{p s}(-1)^{m} Y_{l,-m}\left(\boldsymbol{k}_{v}^{p s}\right) \\
& =\sum_{v} w_{v} \sum_{p, s} \Phi_{v}^{p s} Y_{l, m}^{*}\left(\boldsymbol{k}_{v}^{p s *}\right) .
\end{aligned}
$$

Here, $*$ denotes the complex conjugate. The fact that - in contrast to the nodes - the weights $w_{v}$ do not depend on $p$ nor $s$ has also been used. In the following sections, the accelerated scheme will be developed.

\subsection{Aggregation}

The accelerated scheme relies for a great deal on the connection (17) between the discretization points for the six representations. Substitution of (17) in $\Theta_{v}^{p s}$ yields:

$$
\Theta_{v}^{p s}=\sum_{l=0}^{L} \sum_{m=-l}^{l} a_{l m} j^{l} Y_{l, m}\left(\mathbf{R}_{0}^{-1} \cdot\left[s \mathbf{R}\left(\frac{2 p \pi}{3}, \boldsymbol{u}_{z}\right)\right] \cdot \mathbf{R}_{0} \cdot \boldsymbol{k}_{v}^{3+}\right) .
$$

The outer rotation in the argument of the spherical harmonic can be brought outside the function by means of the Wigner D-matrices, defined in Appendix, as follows

$$
\begin{aligned}
\Theta_{v}^{p s} & =\sum_{l=0}^{L} \sum_{m=-l}^{l} \sum_{m_{1}} a_{l m} j^{l} D_{m, m_{1}}^{l}\left(\mathbf{R}_{0}^{-1}\right) Y_{l, m_{1}}\left(s \mathbf{R}\left(\frac{2 p \pi}{3}, \boldsymbol{u}_{z}\right) \cdot \mathbf{R}_{0} \cdot \boldsymbol{k}_{v}^{3+}\right) \\
& =\sum_{l=0}^{L} \sum_{m_{1}=-l}^{l} a_{l m_{1}}^{\prime} j^{l} Y_{l, m_{1}}\left(s \mathbf{R}\left(\frac{2 p \pi}{3}, \boldsymbol{u}_{z}\right) \cdot \mathbf{R}_{0} \cdot \boldsymbol{k}_{v}^{3+}\right)
\end{aligned}
$$


where

$$
a_{l m_{1}}^{\prime}=\sum_{m} a_{l m} D_{m, m_{1}}^{l}\left(\mathbf{R}_{0}^{-1}\right)
$$

This sum runs over $m$ only and thus can be done fast, compared to the full aggregation. After this operation, all sources are actually rotated to a reference system in which the former $\frac{1}{\sqrt{3}}\left(\boldsymbol{u}_{x}+\boldsymbol{u}_{y}+\boldsymbol{u}_{z}\right)$ axis is the new $z$-axis. In a second step the dependence on $p$ and $s$ can be removed. According to Eq. (A.6) the spherical harmonics are eigenfunctions of the inversion operator (which replaces $\boldsymbol{r}$ by $-\boldsymbol{r}$ ) with eigenvalues $(-1)^{l}$, which allows for the removal of $s$. Moving the $p$-dependence out of the spherical harmonic can again be done using the Wigner D-matrices. However a crucial point here is that Wigner D-matrices for rotations around the $z$-axis are diagonal, as stated in (A.20). This yields:

$$
\Theta_{v}^{p s}=\sum_{l=0}^{L} \sum_{m=-l}^{l} a_{l m}^{\prime} j^{l} s^{l} \mathrm{e}^{\mathrm{i} m \frac{2 p \pi}{3}} Y_{l, m}\left(\mathbf{R}_{0} \cdot \boldsymbol{k}_{v}^{3+}\right)
$$

or after splitting the summations:

$$
\Theta_{v}^{p s}=\sum_{r=0}^{1} \sum_{q=0}^{2} s^{r} \mathrm{e}^{\mathrm{i} q \frac{2 p \pi}{3}} \sum_{r=l m \bmod 2} \sum_{q=m \bmod 3} a_{l m}^{\prime} j^{l} Y_{l, m}\left(\mathbf{R}_{0} \cdot \boldsymbol{k}_{v}^{3+}\right)
$$

The inner summation runs over all $m$ for which $q=m \bmod 3$ and all $l$ for which $r=l \bmod 2$. 'mod' means modulo, so for example $1=7 \bmod 3$. Eq. (26) shows that (18) can be re-expressed as an aggregation towards six new radiation patterns $\kappa_{v}^{q r}$

$$
\kappa_{v}^{q r}=\sum_{r=l \bmod 2} \sum_{q=m \bmod 3} a_{l m}^{\prime} j^{l} Y_{l, m}\left(\mathbf{R}_{0} \cdot \boldsymbol{k}_{v}^{3+}\right) \quad \forall q \in\{0,1,2\}, \quad \forall r \in\{0,1\}
$$

However, only one sixth of the multipoles contributes to each of these patterns. In this way, calculating these six new patterns costs as little as calculating one pattern with the direct formula (18). Of course, there is the extra cost of a postprocessing step, i.e., the summation over $q$ and $r$ in (26), but this cost has only an $\mathcal{O}\left(L^{2}\right)$ complexity and is negligible compared to the entire aggregation. Note that this whole scheme works totally independent from the choice of the discretization points $\boldsymbol{k}_{v}^{3+}$. Only the connection (17) is required. Another important point was brought up by one of the reviewers: very elongated structures require less than six radiation patterns to be computed. For example a long wire will need the MLFMA groups to have only two radiation patterns. In this case (26) will only yield an asymptotic factor two. If an MLFMA group needs only one radiation pattern, then a switch to the direct method should be made. The same considerations apply to the disaggregation and the first method for the vectorial case. The second method will be shown to be capable of accelerating by a factor 2 even if only one radiation pattern is required.

An explicit operation count will be done to show that the proposed method requires less multiplications than the direct approach for most practical cases. The operation count for doing the rotation on $a_{l m}(24)$ is:

$$
\sum_{l=0}^{L}(2 l+1)^{2}=\frac{1}{3}\left(4 L^{3}+12 L^{2}+11 L+3\right)
$$

The operation count for the construction of the new radiation patterns $\kappa_{v}^{q r}$ clearly is $V(L+1)^{2} . V$ is the number of discretization points for the Green function's integral representation. The postprocessing step requires $36 \mathrm{~V}$ multiplications. Hence the total number of multiplications is:

$$
\frac{1}{3}\left(4 L^{3}+12 L^{2}+11 L+3\right)+V\left(L^{2}+2 L+37\right)
$$

The number of multiplications without acceleration is $6 V(L+1)^{2}$. Therefore, the value of $V$ for which the proposed method is faster than the one without acceleration is given by:

$$
V>\frac{1}{3} \frac{4 L^{3}+12 L^{2}+11 L+3}{5 L^{2}+10 L-31} \quad \forall L>1
$$


For the case where $L=1,>$ in Eq. (30) has to be replaced by $<$ because $5 L^{2}+10 L-31$ is negative. Therefore, if $L=1,(30)$ is not satisfied and the new method is slower than the direct approach. For $L=2$, the right hand side becomes $\frac{35}{9}<4$. Since the number of discretization points is always more than 3, (30) is always satisfied. Moreover, $V=\mathcal{O}\left(L^{2}\right)$ because the multipole and plane wave expansion have approximately the same information content. This makes sure that Eq. (30) is also satisfied for larger $L$. Therefore it is safe to say that for any $L>1$, the method presented in 4.2 is faster than the direct approach. It has to be acknowledged that this operation count has to be put in the right perspective, because different optimizations and hardware can have a great influence on performance.

\subsection{Disaggregation}

The disaggregation can also be accelerated. The operations are analogous to the aggregation, but are done in reversed order. A short treatment is given below for completeness. The derivation is again started by using (17) in (20), which yields:

$$
b_{l m}=\sum_{v} w_{v} \sum_{p, s} \Phi_{v}^{p s} Y_{l, m}^{*}\left(\mathbf{R}_{0}^{-1} \cdot\left[s \mathbf{R}\left(\frac{2 p \pi}{3}, \boldsymbol{u}_{z}\right)\right] \cdot \mathbf{R}_{0} \cdot \boldsymbol{k}_{v}^{3+*}\right) .
$$

As a first step it is possible to remove the last rotation by contracting $b_{l m}$ with $D_{m_{2}, m}^{l *}\left(\mathbf{R}_{0}\right)$ :

$$
b_{l m_{2}}^{\prime}=\sum_{m} D_{m_{2}, m}^{l *}\left(\mathbf{R}_{0}\right) b_{l m}=\sum_{v} w_{v} \sum_{p, s} \Phi_{v}^{p s} Y_{l, m_{2}}^{*}\left(s \mathbf{R}\left(\frac{2 p \pi}{3}, \boldsymbol{u}_{z}\right) \cdot \mathbf{R}_{0} \cdot \boldsymbol{k}_{v}^{3+*}\right) .
$$

Again the rotation around the $z$-axis can be taken out of the spherical harmonics by using the diagonal Wigner D-matrices:

$$
\begin{aligned}
b_{l m_{2}}^{\prime} & =\sum_{v} w_{v} \sum_{p, s} \Phi_{v}^{p s} s^{l} \sum_{m_{3}=-l}^{l} D_{m_{2}, m_{3}}^{l *}\left(\mathbf{R}\left(\frac{2 p \pi}{3}, \boldsymbol{u}_{z}\right)\right) Y_{l, m_{3}}^{*}\left(\mathbf{R}_{0} \cdot \boldsymbol{k}_{v}^{3+*}\right) \\
& =\sum_{v} w_{v} \sum_{p, s} \Phi_{v}^{p s} s^{l} \mathrm{e}^{-\mathrm{i} m_{2} \frac{2 p \pi}{3}} Y_{l, m_{2}}^{*}\left(\mathbf{R}_{0} \cdot \boldsymbol{k}_{v}^{3+*}\right) .
\end{aligned}
$$

The summation over $p, s$ gives rise to 6 distinct new disaggregation patterns:

$$
\Xi_{v}^{k, r}=\sum_{p, s} \Phi_{v}^{p s} s^{r} \mathrm{e}^{-\mathrm{i} k \frac{2 p \pi}{3}}, \quad \forall k \in\{0,1,2\}, \quad \in\{0,1\} .
$$

These new patterns allow for a faster disaggregation because they each only contribute to one sixth of the $b_{l m_{1}}^{\prime}$ :

$$
b_{l m_{1}}^{\prime}=\sum_{v} w_{v} \Xi_{v}^{m_{1} \bmod 3, l \bmod 2} Y_{l, m_{1}}\left(\mathbf{R}_{0} \cdot \boldsymbol{k}_{v}^{3+*}\right) .
$$

In order to obtain the $b_{l m}$, the $b_{l m_{1}}^{\prime}$ have only to be rotated back:

$$
\begin{aligned}
b_{l m} & =\sum_{m_{3}=-l}^{l} \sum_{m_{1}=-l}^{l} D_{m, m_{1}}^{l *}\left(\mathbf{R}_{0}^{-1}\right) D_{m_{1}, m_{3}}^{l *}\left(\mathbf{R}_{0}\right) b_{l, m_{3}} \\
& =\sum_{m_{1}=-l}^{l} D_{m, m_{1}}^{l *}\left(\mathbf{R}_{0}^{-1}\right) b_{l, m_{1}}^{\prime} \\
& =\sum_{m_{1}=-l}^{l} D_{m_{1}, m}^{l}\left(\mathbf{R}_{0}\right) b_{l, m_{1}}^{\prime}
\end{aligned}
$$

\section{A faster (dis)aggregation for the vectorial case}

The proposed technique can also be used in the vectorial case, as is needed for the general electromagnetic case. Multipoles for the vectorial case, or vector multipoles, can be collected in two independent sets, which 
will be called $\boldsymbol{M}_{l m}(\gamma \boldsymbol{r})$ and $\boldsymbol{N}_{l m}(\gamma \boldsymbol{r})$ as defined in Appendix. The multipole coefficients associated with these multipoles will thus carry the label $M$ or $N$ besides the usual $l$ and $m$. Because the vectorial case is less well-known, a short derivation of the formulas for the (dis)aggregation will be given here. Let $\boldsymbol{\Phi}(\boldsymbol{r})$ be the vectorial field generated by sources inside a sphere with center $\boldsymbol{r}_{1}$. This field can be expanded into outgoing multipoles:

$$
\boldsymbol{\Phi}(\boldsymbol{r})=\sum_{l=0}^{L} \sum_{m=-l}^{l}\left[a_{l m}^{M} \boldsymbol{M}_{l, m}^{h^{(2)}}\left(\gamma\left(\boldsymbol{r}-\boldsymbol{r}_{1}\right)\right)+a_{l m}^{N} \boldsymbol{N}_{l, m}^{h^{(2)}}\left(\gamma\left(\boldsymbol{r}-\boldsymbol{r}_{1}\right)\right)\right]
$$

$\boldsymbol{\Phi}(\boldsymbol{r})$ can now be expanded around $\boldsymbol{r}_{2}$ as:

$$
\boldsymbol{\Phi}(\boldsymbol{r})=\sum_{l=0}^{L} \sum_{m=-l}^{l}\left[b_{l m}^{M} \boldsymbol{M}_{l, m}^{j}\left(\gamma\left(\boldsymbol{r}-\boldsymbol{r}_{2}\right)\right)+b_{l m}^{N} \boldsymbol{N}_{l, m}^{j}\left(\gamma\left(\boldsymbol{r}-\boldsymbol{r}_{2}\right)\right)\right]
$$

with $b_{l, m}^{M}$ and $b_{l, m}^{N}$ defined by means of the multipole translation matrix [13]:

$$
\left[\begin{array}{c}
\boldsymbol{b}^{M} \\
\boldsymbol{b}^{N}
\end{array}\right]=\boldsymbol{\alpha}^{\mathrm{vec}}(\gamma \boldsymbol{r}) \cdot\left[\begin{array}{c}
\boldsymbol{a}^{M} \\
\boldsymbol{a}^{N}
\end{array}\right]=\left[\begin{array}{ll}
\boldsymbol{\alpha}^{M M}(\gamma \boldsymbol{r}) & \boldsymbol{\alpha}^{N M}(\gamma \boldsymbol{r}) \\
\boldsymbol{\alpha}^{N M}(\gamma \boldsymbol{r}) & \boldsymbol{\alpha}^{M M}(\gamma \boldsymbol{r})
\end{array}\right] \cdot\left[\begin{array}{c}
\boldsymbol{a}^{M} \\
\boldsymbol{a}^{N}
\end{array}\right]
$$

with

$$
\begin{aligned}
& {\left[\boldsymbol{\alpha}^{M M}(\gamma \boldsymbol{r})\right]_{l^{\prime}, m^{\prime}, l, m}=2 j^{l-l^{\prime}} \int_{-\infty}^{\infty} \int_{-\infty}^{\infty} \boldsymbol{X}_{l, m}(\boldsymbol{k}) \cdot\left[\boldsymbol{X}_{l^{\prime}, m^{\prime}}\left(\boldsymbol{k}^{*}\right)\right]^{*} \mathrm{e}^{-\mathrm{j} \boldsymbol{k} \cdot \boldsymbol{r}} \frac{\mathrm{d} k_{x} \mathrm{~d} k_{y}}{\gamma k_{z}(\gamma, K)}} \\
& {\left[\boldsymbol{\alpha}^{M N}(\gamma \boldsymbol{r})\right]_{l^{\prime}, m^{\prime}, l, m}=2 j^{l-l^{\prime}} \int_{-\infty}^{\infty} \int_{-\infty}^{\infty}\left[-\mathrm{j} \boldsymbol{\Psi}_{l, m}(\boldsymbol{k})\right] \cdot\left[\boldsymbol{X}_{l^{\prime}, m^{\prime}}\left(\boldsymbol{k}^{*}\right)\right]^{*} \mathrm{e}^{-\mathrm{j} \boldsymbol{k} \cdot \boldsymbol{r}} \frac{\mathrm{d} k_{x} \mathrm{~d} k_{y}}{\gamma k_{z}(\gamma, K)}}
\end{aligned}
$$

Here, the $\boldsymbol{X}_{l, m}(\cdot)$ and $\boldsymbol{\Psi}_{l, m}(\cdot)$ are the so-called vector spherical harmonics, again defined in Appendix. Integrals (43) and (44) can again be split up in a propagating and an evanescent part and discretized. The propagating part will again be omitted. By means of (43) and (44), and the formulas in Appendix (A.15) and (A.16), the aggregation toward the six evanescent radiation patterns can be written as follows:

$$
\boldsymbol{\Theta}_{v}^{p s}=\sum_{l=0}^{L} \sum_{m=-l}^{l} j^{l}\left[a_{l m}^{M} \boldsymbol{X}_{l, m}\left(\boldsymbol{k}_{v}^{p s}\right)-\mathrm{j} a_{l m}^{N} \boldsymbol{\Psi}_{l, m}\left(\boldsymbol{k}_{v}^{p s}\right)\right]
$$

The vector $\Theta_{v}^{p s}$ has three components. From the definition of the vector spherical harmonics in Appendix, it is clear that $\boldsymbol{\Theta}_{v}^{p s} \cdot \boldsymbol{k}_{v}^{p s}=0$. This relation removes one degree of freedom and permits representing the vector $\boldsymbol{\Theta}_{v}^{p s}$ with only the two components orthogonal to $\boldsymbol{k}_{v}^{p s}$. An obvious choice for these components is to take the part of $\boldsymbol{\Theta}_{v}^{p s}$ parallel with $\boldsymbol{u}_{\phi, v}^{p s}$ and $\boldsymbol{u}_{\theta, v}^{p s}$, which are defined as follows:

$$
\begin{aligned}
\boldsymbol{u}_{\phi, v}^{p s} & =\mathbf{R}_{0}^{-1} \cdot \mathbf{R}\left(\frac{2 p \pi}{3}, \boldsymbol{u}_{z}\right) \cdot \mathbf{R}_{0} \cdot \boldsymbol{u}_{\phi, v}^{3+} \\
\boldsymbol{u}_{\theta, v}^{p s} & =s \mathbf{R}_{0}^{-1} \cdot \mathbf{R}\left(\frac{2 p \pi}{3}, \boldsymbol{u}_{z}\right) \cdot \mathbf{R}_{0} \cdot \boldsymbol{u}_{\theta, v}^{3+}
\end{aligned}
$$

Here $\boldsymbol{u}_{\phi, v}^{3+}$ and $\boldsymbol{u}_{\theta, v}^{3+}$ are the two usual nonradial unit vectors in the spherical coordinate system. Expression (46) does not contain an $s$ in the right hand side for reasons that will be explained in Section 4.2. The aggregation to $\boldsymbol{\Theta}_{v}^{p s}$ is thus reduced to an aggregation to two scalar quantities namely to $\boldsymbol{u}_{\phi, v}^{p s} \cdot \boldsymbol{\Theta}_{v}^{p s}$ and $\boldsymbol{u}_{\theta, v}^{p s} \cdot \boldsymbol{\Theta}_{v}^{p s}$ :

$$
\begin{gathered}
\boldsymbol{u}_{\phi, v}^{p s} \cdot \boldsymbol{\Theta}_{v}^{p s}=\sum_{l=0}^{L} \sum_{m=-l}^{l} j^{l}\left[a_{l m}^{M} \boldsymbol{u}_{\phi, v}^{p s} \cdot \boldsymbol{X}_{l, m}\left(\boldsymbol{k}_{v}^{p s}\right)-\mathrm{j} a_{l m}^{N} \boldsymbol{u}_{\phi, v}^{p s} \cdot \boldsymbol{\Psi}_{l, m}\left(\boldsymbol{k}_{v}^{p s}\right)\right] \\
\boldsymbol{u}_{\theta, v}^{p s} \cdot \boldsymbol{\Theta}_{v}^{p s}=\sum_{l=0}^{L} \sum_{m=-l}^{l} j^{l}\left[a_{l m}^{M} \boldsymbol{u}_{\theta, v}^{p s} \cdot \boldsymbol{X}_{l, m}\left(\boldsymbol{k}_{v}^{p s}\right)-\mathrm{j} a_{l m}^{N} \boldsymbol{u}_{\theta, v}^{p s} \cdot \boldsymbol{\Psi}_{l, m}\left(\boldsymbol{k}_{v}^{p s}\right)\right]
\end{gathered}
$$

This aggregation for the vectorial case can now be accelerated in two ways. These will both be outlined below. In what follows we will only consider (48) because the formulas for (49) can be derived in a very similar fashion. 


\subsection{A faster aggregation for the vectorial case: method 1}

The rotations of the argument of the vector spherical harmonics can again be brought outside by means of the Wigner D-matrices which yields:

$$
\begin{aligned}
\boldsymbol{u}_{\phi, v}^{p s} \cdot \boldsymbol{\Theta}_{v}^{p s}= & \sum_{l=0}^{L} \sum_{m=-l}^{l}\left[\left[\mathbf{R}\left(\frac{2 p \pi}{3}, \boldsymbol{u}_{z}\right) \cdot \mathbf{R}_{0} \cdot \boldsymbol{u}_{\phi, v}^{3+}\right] \cdot \boldsymbol{X}_{l, m}\left(s \mathbf{R}\left(\frac{2 p \pi}{3}, \boldsymbol{u}_{z}\right) \cdot \mathbf{R}_{0} \cdot \boldsymbol{k}_{v}^{3+}\right) a_{l m}^{\prime M}-\mathrm{j}\left[\mathbf{R}\left(\frac{2 p \pi}{3}, \boldsymbol{u}_{z}\right) \cdot \mathbf{R}_{0} \cdot \boldsymbol{u}_{\phi, v}^{3+}\right]\right. \\
& \left.\cdot \boldsymbol{\Psi}_{l, m}\left(s \mathbf{R}\left(\frac{2 p \pi}{3}, \boldsymbol{u}_{z}\right) \cdot \mathbf{R}_{0} \cdot \boldsymbol{k}_{v}^{3+}\right) a_{l m}^{\prime N}\right]
\end{aligned}
$$

with:

$$
\begin{aligned}
& a_{l m_{1}}^{\prime M}=\sum_{m} D_{m, m_{1}}^{l}\left(\mathbf{R}_{0}^{-1}\right) j^{l} a_{l, m}^{M} \\
& a_{l m_{1}}^{\prime N}=\sum_{m} D_{m, m_{1}}^{l}\left(\mathbf{R}_{0}^{-1}\right) j^{l} a_{l, m}^{N}
\end{aligned}
$$

To remove the dependence on $s$ the properties of the vector spherical harmonics (A.13) and (A.14) can be used. The dependence on $p$ can again be removed using the diagonal Wigner D-matrices for rotations around the $z$-axis:

$$
\begin{aligned}
\boldsymbol{u}_{\phi, v}^{p s} \cdot \boldsymbol{\Theta}_{v}^{p s}= & \sum_{l=0}^{L} \sum_{m=-l}^{l} s^{l} \mathrm{e}^{\mathrm{i} m \frac{2 p \pi}{3}}\left[\left(\mathbf{R}_{0} \cdot \boldsymbol{u}_{\phi, v}^{3+}\right) \cdot \boldsymbol{X}_{l, m}\left(\mathbf{R}_{0} \cdot \boldsymbol{k}_{v}^{3+}\right) a_{l m}^{\prime M}\right. \\
& \left.-\mathrm{j} s\left(\mathbf{R}_{0} \cdot \boldsymbol{u}_{\phi, v}^{3+}\right) \cdot \boldsymbol{\Psi}_{l, m}\left(\mathbf{R}_{0} \cdot \boldsymbol{k}_{v}^{3+}\right) a_{l m}^{\prime N}\right]
\end{aligned}
$$

or after splitting the summations:

$$
\boldsymbol{u}_{\phi, v}^{p s} \cdot \boldsymbol{\Theta}_{v}^{p s}=\sum_{r=0}^{1} \sum_{q=0}^{2} s^{r} \mathrm{e}^{\mathrm{i} q \frac{2 p \pi}{3}} \sum_{r=l \bmod 2} \sum_{q=m \bmod 3}\left[\left(\mathbf{R}_{0} \cdot \boldsymbol{u}_{\phi, v}^{3+}\right) \cdot \boldsymbol{X}_{l, m}\left(\mathbf{R}_{0} \cdot \boldsymbol{k}_{v}^{3+}\right) a_{l m}^{\prime M}-\mathrm{j} s\left(\mathbf{R}_{0} \cdot \boldsymbol{u}_{\phi, v}^{3+}\right) \cdot \Psi_{l, m}\left(\mathbf{R}_{0} \cdot \boldsymbol{k}_{v}^{3+}\right) a_{l m}^{\prime N}\right]
$$

Despite the splitting of the sums, there is still an $s$ inside the brackets. This can be resolved by rewriting the sum as:

$$
\boldsymbol{u}_{\phi, v}^{p s} \cdot \boldsymbol{\Theta}_{v}^{p s}=\sum_{r=0}^{1} \sum_{q=0}^{2} s^{r} \mathrm{e}^{\mathrm{i} q^{2 p \pi}} \sum_{q=m \bmod 3}\left[\sum_{r=l \bmod 2}\left(\mathbf{R}_{0} \cdot \boldsymbol{u}_{\phi, v}^{3+}\right) \cdot \boldsymbol{X}_{l, m}\left(\mathbf{R}_{0} \cdot \boldsymbol{k}_{v}^{3+}\right) a_{l m}^{\prime M}-\mathrm{j} \sum_{l=(1-r) \bmod 2}\left(\mathbf{R}_{0} \cdot \boldsymbol{u}_{\phi, v}^{3+}\right) \cdot \boldsymbol{\Psi}_{l, m}\left(\mathbf{R}_{0} \cdot \boldsymbol{k}_{v}^{3+}\right) a_{l m}^{\prime N}\right]
$$

Eq. (56) again requires only one sixth of the amount of work that is needed for (48). Indeed, there are six new radiation patterns, but each of them requires six times less work. The disaggregation is entirely analogous to the aggregation.

\subsection{A faster aggregation for the vectorial case: Method 2}

The (dis)aggregation in the vectorial case can also be accelerated in a different way. This method does not use the inversion properties of the vector spherical harmonics, but rather uses the transformation properties under the curl operator. This method is easier to implement and slightly faster than method 1 . The aggregation, given by (48) and (49), can be concisely written as:

$$
\left[\begin{array}{c}
\boldsymbol{u}_{\phi, v}^{p s} \cdot \boldsymbol{\Theta}_{v}^{p s} \\
\boldsymbol{u}_{\theta, v}^{p s} \cdot \Theta_{v}^{p s}
\end{array}\right]=\sum_{l=0}^{L} \sum_{m=-l}^{l}\left[\begin{array}{ll}
A_{\phi, v}^{M, p, s, l, m} & A_{\phi, v}^{N, p, s, l, m} \\
A_{\theta, v}^{M, p, s, l, m} & A_{\theta, v}^{N, p, s, l, m}
\end{array}\right] \cdot\left[\begin{array}{c}
a_{l, m}^{M} \\
a_{l, m}^{N}
\end{array}\right]
$$


with:

$$
\begin{aligned}
& A_{\theta, v}^{M, p, s, l, m}=j^{l} \boldsymbol{u}_{\theta, v}^{p s} \cdot \boldsymbol{X}_{l, m}\left(\boldsymbol{k}_{v}^{p s}\right), \\
& A_{\theta, v}^{N, p, s, l, m}=j^{l} \boldsymbol{u}_{\theta, v}^{p s} \cdot\left[-\mathrm{j} \boldsymbol{\Psi}_{l, m}\left(\boldsymbol{k}_{v}^{p s}\right)\right], \\
& A_{\phi, v}^{M, p, s, l, m}=j^{l} \boldsymbol{u}_{\phi, v}^{p s} \cdot \boldsymbol{X}_{l, m}\left(\boldsymbol{k}_{v}^{p s}\right), \\
& A_{\phi, v}^{N, p, s, l, m}=j^{l} \boldsymbol{u}_{\phi, v}^{p s} \cdot\left[-\mathrm{j} \Psi_{l, m}\left(\boldsymbol{k}_{v}^{p s}\right)\right] .
\end{aligned}
$$

As stated before, the unit vectors $\boldsymbol{u}_{\theta, v}^{p s}$ and $\boldsymbol{u}_{\phi, v}^{p s}$ are orthogonal to each other and to $\boldsymbol{k}_{v}^{p s}$. As a consequence:

$$
\begin{aligned}
& \boldsymbol{u}_{\theta, v}^{p s} \times\left(\boldsymbol{k}_{v}^{p s} \times \boldsymbol{u}_{\phi, v}^{p s}\right)=\boldsymbol{k}_{v}^{p s}\left(\boldsymbol{u}_{\theta, v}^{p s} \cdot \boldsymbol{u}_{\phi, v}^{p s}\right)-\boldsymbol{u}_{\phi, v}^{p s}\left(\boldsymbol{u}_{\theta, v}^{p s} \cdot \boldsymbol{k}_{v}^{p s}\right) \\
& \boldsymbol{u}_{\theta, v}^{p s} \times\left(\boldsymbol{k}_{v}^{p s} \times \boldsymbol{u}_{\phi, v}^{p s}\right)=0
\end{aligned}
$$

which yields, with the fact that $\boldsymbol{u}_{\phi, v}^{p s}$ and $\boldsymbol{u}_{\theta, v}^{p s}$ have unit length:

$$
\boldsymbol{k}_{v}^{p s} \times \boldsymbol{u}_{\phi, v}^{p s}= \pm \gamma \boldsymbol{u}_{\theta, v}^{p s}
$$

The sign can be chosen freely, but it is convenient to work with a plus sign, since that is similar to the usual unit vectors in spherical coordinates. As a consequence:

$$
\begin{gathered}
\boldsymbol{k}_{v}^{p s} \times \boldsymbol{u}_{\phi, v}^{p s}=\gamma \boldsymbol{u}_{\theta, v}^{p s} \\
\boldsymbol{u}_{\theta, v}^{p s} \times \boldsymbol{k}_{v}^{p s}=\gamma \boldsymbol{u}_{\phi, v}^{p s} \\
\gamma \boldsymbol{u}_{\phi, v}^{p s} \times \boldsymbol{u}_{\theta, v}^{p s}=\boldsymbol{k}_{v}^{p s}
\end{gathered}
$$

This particular choice explains why $\boldsymbol{u}_{\theta, v}^{p s}$ has the additional factor $s$ in (47):

$$
\begin{aligned}
\boldsymbol{k}_{v}^{p s} \times \boldsymbol{u}_{\phi, v}^{p s} & =\gamma \boldsymbol{u}_{\theta, v}^{p s}=\mathbf{R}_{0}^{-1} \cdot \mathbf{R}\left(\frac{2 p \pi}{3}, \boldsymbol{u}_{z}\right) \cdot \mathbf{R}_{0} \cdot\left[s \boldsymbol{k}_{v}^{3+} \times \boldsymbol{u}_{\phi, v}^{3+}\right] \\
& =s \gamma \mathbf{R}_{0}^{-1} \cdot \mathbf{R}\left(\frac{2 p \pi}{3}, \boldsymbol{u}_{z}\right) \cdot \mathbf{R}_{0} \cdot \boldsymbol{u}_{\theta, v}^{3+}
\end{aligned}
$$

With this choice, it can be easily shown that:

$$
\begin{aligned}
& A_{\phi, v}^{M, p, s, l, m}=-j^{l}\left(\frac{\boldsymbol{k}_{v}^{p s}}{\gamma} \times \boldsymbol{u}_{\theta, v}^{p s}\right) \cdot \boldsymbol{X}_{l, m}\left(\boldsymbol{k}_{v}^{p s}\right)=j^{l} \boldsymbol{u}_{\theta, v}^{p s} \cdot \boldsymbol{\Psi}_{l, m}\left(\boldsymbol{k}_{v}^{p s}\right)=\mathrm{j} A_{\theta, v}^{N, p, s, l, m} \\
& A_{\phi, v}^{N, p, s, l, m}=j^{l+1}\left(\frac{\boldsymbol{k}_{v}^{p s}}{\gamma} \times \boldsymbol{u}_{\theta, v}^{p s}\right) \cdot \boldsymbol{\Psi}_{l, m}\left(\boldsymbol{k}_{v}^{p s}\right)=j^{l+1} \boldsymbol{u}_{\theta, v}^{p s} \cdot \boldsymbol{X}_{l, m}\left(\boldsymbol{k}_{v}^{p s}\right)=\mathrm{j} A_{\theta, v}^{M, p, s, l, m}
\end{aligned}
$$

So (57) becomes:

$$
\left[\begin{array}{c}
-\mathrm{j} \boldsymbol{u}_{\phi, v}^{p s} \cdot \boldsymbol{\Theta}_{v}^{p s} \\
\boldsymbol{u}_{\theta, v}^{p s} \cdot \boldsymbol{\Theta}_{v}^{p s}
\end{array}\right]=\sum_{l=0}^{L} \sum_{m=-l}^{l}\left[\begin{array}{cc}
A_{\theta, v}^{N, p, s, l, m} & A_{\theta, v}^{M, p, s, l, m} \\
A_{\theta, v}^{M, p, s, l, m} & A_{\theta, v}^{N, p, s, l, m}
\end{array}\right] \cdot\left[\begin{array}{c}
a_{l, m}^{M} \\
a_{l, m}^{N}
\end{array}\right]
$$

It is worthwhile to point out that the $2 \times 2$ matrix occurring in (72) is circulant and can thus be diagonalized by means of the $2 \times 2$ Fourier matrix. After some calculations, this yields:

$$
\left[\begin{array}{c}
\left(-\mathrm{j} \boldsymbol{u}_{\phi, v}^{p s}+\boldsymbol{u}_{\theta, v}^{p s}\right) \cdot \boldsymbol{\Theta}_{v}^{p s} \\
\left(-\mathrm{j} \boldsymbol{u}_{\phi, v}^{p s}-\boldsymbol{u}_{\theta, v}^{p s}\right) \cdot \boldsymbol{\Theta}_{v}^{p s}
\end{array}\right]=\frac{1}{2} \sum_{l=0}^{L} \sum_{m=-l}^{l}\left[\begin{array}{cc}
A_{\theta, v}^{N, p, s, l, m}+A_{\theta, v}^{M, p, s, l, m} & 0 \\
0 & A_{\theta, v}^{N, p, s, l, m}-A_{\theta, v}^{M, p, s, l, m}
\end{array}\right] \cdot\left[\begin{array}{l}
a_{l, m}^{M}+a_{l, m}^{N} \\
a_{l, m}^{M}-a_{l, m}^{N}
\end{array}\right]
$$

This expression allows to reduce the amount of work associated with the aggregations by a factor two. It also exposes some fundamental aspects of vector multipoles. For example consider the vector multipole translation matrix $\boldsymbol{\alpha}^{\mathrm{vec}}(\gamma \boldsymbol{r})$ as given in (42). This matrix $\boldsymbol{\alpha}^{\mathrm{vec}}(\gamma \boldsymbol{r})$ is block-circulant and can thus also be block-diagonalized by means of the $2 \times 2$ Fourier matrix. This yields:

$$
\left[\begin{array}{l}
\boldsymbol{b}^{M}+\boldsymbol{b}^{N} \\
\boldsymbol{b}^{M}-\boldsymbol{b}^{N}
\end{array}\right]=\left[\begin{array}{cc}
\boldsymbol{\alpha}^{M M}+\boldsymbol{\alpha}^{N M} & 0 \\
0 & \boldsymbol{\alpha}^{M M}-\boldsymbol{\alpha}^{N M}
\end{array}\right] \cdot\left[\begin{array}{c}
\boldsymbol{a}^{M}+\boldsymbol{a}^{N} \\
\boldsymbol{a}^{M}-\boldsymbol{a}^{N}
\end{array}\right]
$$


This allows a two times faster application of the vector multipole translation matrix. Another example can be found in [14]: the recurrences for the calculation of the vector multipole translation matrices $\alpha^{M M}$ and $\alpha^{M N}$ are coupled, but they can be decoupled by using $\alpha^{M M} \pm \alpha^{M N}$ instead. As for the vector multipoles themselves, this block-diagonalization of $\alpha^{\mathrm{vec}}(\gamma \boldsymbol{r})$ is a change of basis which boils down to using using the combinations $\boldsymbol{M}_{l m}^{f} \pm \boldsymbol{N}_{l m}^{f}$ instead of $\boldsymbol{M}_{l m}^{f}$ and $\boldsymbol{N}_{l m}^{f}$. All this is caused by the transformation properties of $\boldsymbol{M}_{l m}^{f} \pm \boldsymbol{N}_{l m}^{f}$ under the curl operator:

$$
\boldsymbol{\nabla} \times\left[\boldsymbol{M}_{l m}^{f} \pm \boldsymbol{N}_{l m}^{f}\right]=\gamma\left[\boldsymbol{N}_{l m}^{f} \pm \boldsymbol{M}_{l m}^{f}\right]= \pm \gamma\left[\boldsymbol{M}_{l m}^{f} \pm \boldsymbol{N}_{l m}^{f}\right]
$$

Apparently $\boldsymbol{M}_{l m}^{f}+\boldsymbol{N}_{l m}^{f}$ and $\boldsymbol{M}_{l m}^{f}-\boldsymbol{N}_{l m}^{f}$ are eigenfunctions of the curl operator with eigenvalues $+\gamma$ and $-\gamma$, respectively. Any electromagnetic field in a source-free region is a superposition of vector multipoles $\boldsymbol{M}_{l m}^{j}$ and $\boldsymbol{N}_{l m}^{j}$ and can thus be decomposed into a part with eigenvalue $+\gamma$ and a part with eigenvalue $-\gamma$ with respect to the curl operator. This is the so-called Beltrami-decomposition of the electromagnetic field $[15,16]$. Because the curl operator commutes with the translation operator, these parts will remain separated under translation. Indeed, applying the curl before or after translation of one of the parts must yield the same result, so no mixing of the two parts can take place under translation. Therefore, the vector multipole translation matrix cannot contain coupling between $\boldsymbol{M}_{l m}^{f}+\boldsymbol{N}_{l m}^{f}$ and $\boldsymbol{M}_{l m}^{f}-\boldsymbol{N}_{l m}^{f}$ fields. For the aggregation, no mixing will occur if the plane waves (to which the aggregation is done) are also eigenfunctions of the curl operator. Although not explicitly visible, this is already the case in (73). To show this, (73) is rewritten as:

$$
\left(-\mathrm{j} \boldsymbol{u}_{\phi, v}^{p s} \pm \boldsymbol{u}_{\theta, v}^{p s}\right) \cdot \boldsymbol{\Theta}_{v}^{p s}=\frac{1}{4} \sum_{l=0}^{L} \sum_{m=-l}^{l} j^{l}\left(-\mathrm{j} \boldsymbol{u}_{\phi, v}^{p s} \pm \boldsymbol{u}_{\theta, v}^{p s}\right) \cdot\left[\boldsymbol{X}_{l, m}\left(\boldsymbol{k}_{v}^{p s}\right) \mp \mathrm{j} \boldsymbol{\Psi}_{l, m}\left(\boldsymbol{k}_{v}^{p s}\right)\right]\left[a_{l, m}^{M} \pm a_{l, m}^{N}\right]
$$

From this it is seen that the plane waves have the form

$$
\left[\boldsymbol{X}_{l, m}\left(\boldsymbol{k}_{v}^{p s}\right) \mp \mathrm{j} \boldsymbol{\Psi}_{l, m}\left(\boldsymbol{k}_{v}^{p s}\right)\right] \mathrm{e}^{-\mathrm{j} \boldsymbol{k}_{v}^{p s} \cdot \boldsymbol{r}}
$$

and it can be verified easily that these plane waves are eigenfunctions of the curl operator:

$$
\boldsymbol{\nabla} \times\left[\left[\boldsymbol{X}_{l, m}\left(\boldsymbol{k}_{v}^{p s}\right) \mp \mathrm{j} \boldsymbol{\Psi}_{l, m}\left(\boldsymbol{k}_{v}^{p s}\right)\right] \mathrm{e}^{-\mathrm{j} \boldsymbol{k}_{v}^{p s} \cdot \boldsymbol{r}}\right]= \pm \gamma\left[\boldsymbol{X}_{l, m}\left(\boldsymbol{k}_{v}^{p s}\right) \mp \mathrm{j} \boldsymbol{\Psi}_{l, m}\left(\boldsymbol{k}_{v}^{p s}\right)\right] \mathrm{e}^{-\mathrm{j} \boldsymbol{k}_{v}^{p s} \cdot \boldsymbol{r}}
$$

A similar block-diagonalization as the one for the aggregation can also be done for the disaggregation, yielding an acceleration by a factor two. The dependencies (17) can now be used again to obtain further acceleration by a factor three, resulting in a total acceleration by a factor six. Unfortunately, the inversion properties (A.13) and (A.14) of the vector harmonics cannot be used any more because $\boldsymbol{M}_{l m}^{f} \pm \boldsymbol{N}_{l m}^{f}$ are not eigenfunctions of the inversion operator. Indeed, the inversion and curl operator do not commute, so their eigenfunctions cannot be the same.

The method described here is slightly faster than the method from Section 4.1. This is because the factor two from the block-diagonalization of the (dis)aggregation is almost completely free of any overhead. Just combine the $a_{l, m}^{M}$ and $a_{l, m}^{N}$ into $a_{l, m}^{M} \pm a_{l, m}^{N}$ before the start of the entire MLFMA and the two sets of coefficients remain completely independent until the FMM is finished. This also permits running the MLFMA on two processors without any communication between them, with perfect load balancing. Moreover, the second method is easier to implement. It is worthwhile to point out that the gain from using eigenfunctions of the curl operator is not limited to evanescent wave solvers. In fact it can be used in any vectorial MLFMA in electromagnetics, including the plane wave method (faster (dis)aggregation if both electric and magnetic currents are present) and LF-MLFMAs (all translation matrices become block-diagonal, as shown in (74), yielding a factor two). Finally, in the method described here the $\boldsymbol{k}_{v}^{p+}$ and $\boldsymbol{k}_{v}^{p-}$ radiation patterns do not couple. So if for example only radiation pattern $\boldsymbol{k}_{v}^{2+}$ is required, only the three $\boldsymbol{k}_{v}^{p+}$ patterns will be calculated. As a consequence an asymptotic factor 2 is still gained compared to the direct method.

An operation count as in Section 3 will now be performed. The operation count for doing the rotation on the two sets of coefficients is:

$$
2 \sum_{l=1}^{L}(2 l+1)^{2}=\frac{2}{3}\left(4 L^{3}+12 L^{2}+11 L\right)
$$


The operation count for the construction of the new radiation patterns clearly is $4 V\left(L^{2}+2 L\right)$. The postprocessing step requires $36 \mathrm{~V}$ multiplications. The total cost is thus:

$$
\frac{2}{3}\left(4 L^{3}+12 L^{2}+11 L\right)+4 V\left(L^{2}+2 L+9\right)
$$

The cost without acceleration is $24 V\left(L^{2}+2 L\right)$. Therefore, the value of $V$ for which the proposed method is faster than the one without acceleration is given by:

$$
V>\frac{L}{6} \frac{4 L^{2}+12 L+11}{5 L^{2}+10 L-9}
$$

Eq. (81) is always satisfied if $L<7$, because the right hand side is certainly smaller than 1 which is smaller than $V$. Moreover, $V=\mathcal{O}\left(L^{2}\right)$ again assures that (81) is also satisfied for larger $L$. Therefore it is safe to say that for any $L$, the method presented in 4.2 is faster than the direct approach. This will also be validated in Section 6 . The acceleration factor is given by:

$$
\frac{24 V\left(L^{2}+4 L\right)}{\frac{3}{2}\left(4 L^{3}+12 L^{2}+11 L\right)+4 V\left(L^{2}+4 L+9\right)}
$$

Again for large $L, V=\mathcal{O}\left(L^{2}\right)$ so the acceleration factor becomes six.

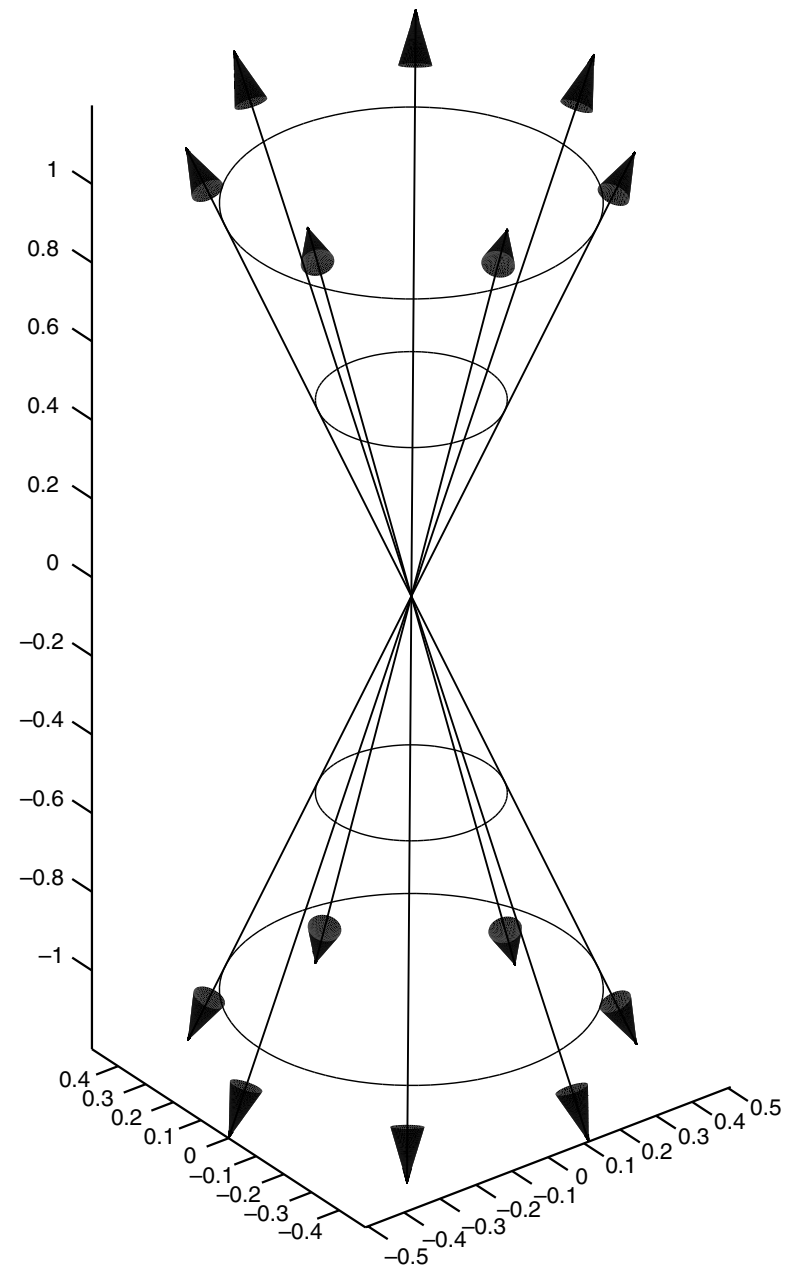

Fig. 3. An example configuration with seven axes. 


\section{Extension to $N$ axes}

The rotation method described in the above can be generalized to a case where $N$ axes are located on a cone as in Fig. 3. This generalization will be demonstrated for the scalar case only, but evidently it can be used for the vectorial case as well. The formula for $\boldsymbol{k}_{v}^{p s}$ becomes

$$
\boldsymbol{k}_{v}^{p s}=\mathbf{R}_{0}^{-1} \cdot\left[s \mathbf{R}\left(\frac{2 p \pi}{N}, \boldsymbol{u}_{z}\right)\right] \cdot \mathbf{R}_{0} \cdot \boldsymbol{k}_{v}^{N+} \quad \forall s \in\{-1,+1\} \quad \forall p \in\{1, \ldots, N\}
$$

with $\mathbf{R}_{0}$ the rotation matrix which rotates the reference frame to a new frame in which the symmetry axis of the cone is the new $z$-axis. When all the steps of the method are repeated, the following expression is obtained:

$$
\Theta_{v}^{p s}=\sum_{r=0}^{1} \sum_{q=0}^{N} s^{r} \mathrm{e}^{\mathrm{i} q \frac{2 p \pi}{N}} \Gamma_{v}^{r q}
$$

Here, $\Gamma_{v}^{r q}$ are $2 N$ new radiation patterns, which each require $2 N$ times less work than one 'normal' pattern. Therefore the total amount of work for evaluating the $\Gamma_{v}^{r q}$ is independent of the number of axes $N$. The recombination step is the only remaining step and can be done with an FFT. Indeed, the sum over $q$ represents precisely an inverse FFT.

\section{Results}

The vectorial stable plane wave method was applied to the scattering from spheres [17]. In particular the Tmatrix method was used, described in detail in [18], which was then accelerated with the vectorial stable plane wave method. The multipole-to-plane-wave and plane-wave-to-multipole operations are natural parts of this algorithm. A uniform discretization of the evanescent integral in both of the integration variables was used. This technique is discussed briefly in [6]. The method from Section 4.2 was used to speed up the (dis)aggregation. Fig. 4 shows the acceleration factor of the (dis)aggregation from the multipole sources on the spheres to the evanescent plane wave radiation patterns of the lowest-level boxes as a function of the multipole order $L$ used on the spheres. The acceleration factor is defined here as the time needed for the direct scheme ((49) and (48)) divided by

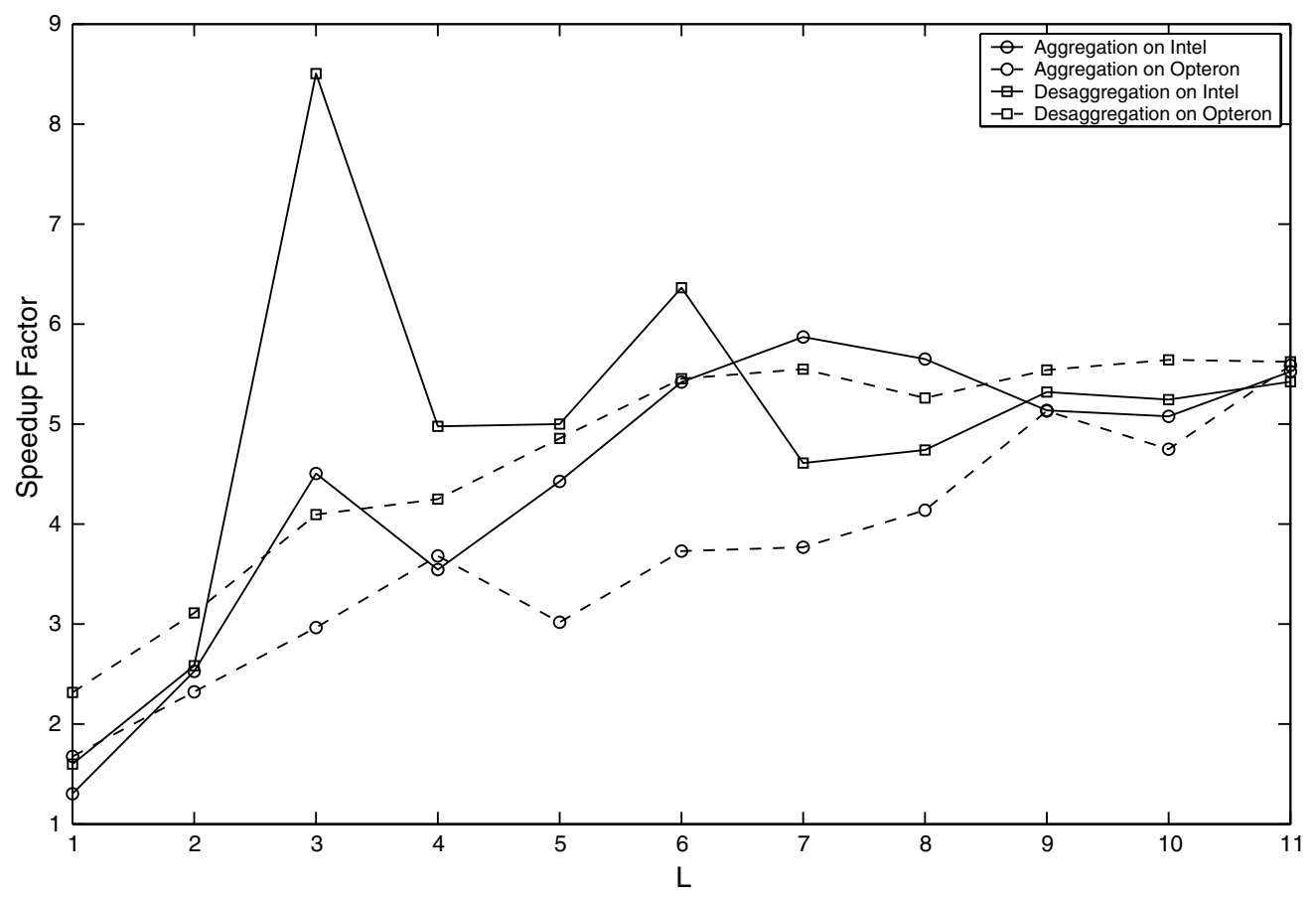

Fig. 4. The acceleration factor of Method 2 for the vectorial case as a function of $L$. The Intel processor is a Pentium $4,2.40 \mathrm{GHz}$ and the other processor is an Opteron 270. 


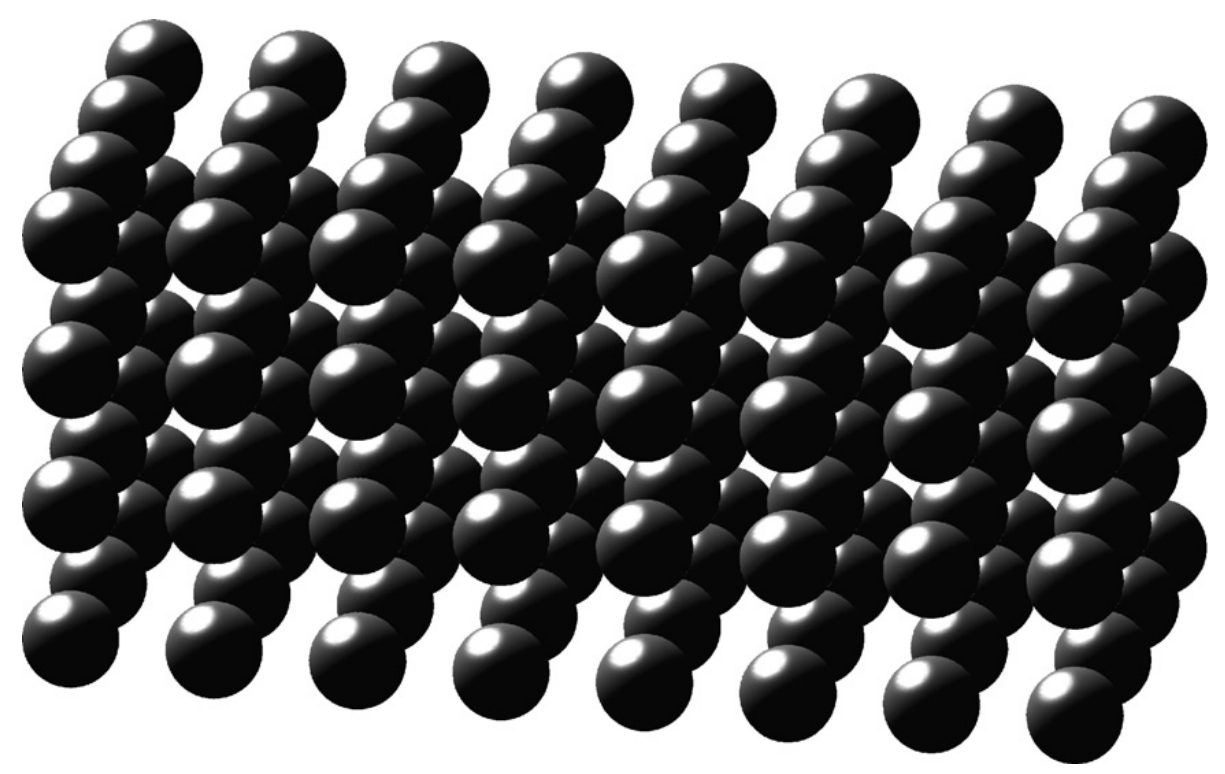

Fig. 5. The geometry for testing the performance of the new method in the stable plane wave method. The spheres with radius $5 \mathrm{~cm}$ and permittivity 12 are located on a $8 \times 4 \times 4$ grid with period $15 \mathrm{~cm}$. The frequency is $0.5 \mathrm{GHz}$.

the time needed for the newly proposed method from Section 4.2. As can be seen, the curves show strong fluctuations. This is caused by pecularities in the hardware because when the code is run on two different processors (an Intel Pentium 4, $2.40 \mathrm{GHz}$ and an AMD Opteron 270), the curves behave differently. However, it is clear that the acceleration factor grows towards six with increasing multipole order for all the curves.

In order to assess the acceleration directly in the context of the stable plane wave method, a typical multiple scattering problem was solved. The problem consists of $8 \times 4 \times 4=128$ spheres with radius $5 \mathrm{~cm}$ on a rectangular grid with period $15 \mathrm{~cm}$. The spheres have a relative permittivity of 12 and a relative permeability of 1 . Fig. 5 shows the geometry of the problem. This scattering problem was solved for various accuracies at a frequency of $0.5 \mathrm{GHz}$, so the aggregation is in the LF regime. The accuracy setting has an influence on both the stable plane wave method and on the multipole order that is necessary to represent the fields on the spheres. In this test, for every multipole order $L$ between 1 and 9, the accuracy obtained by an exact solver was measured, and the stable plane wave method was set to deliver this accuracy. For each box, all the radiation patterns

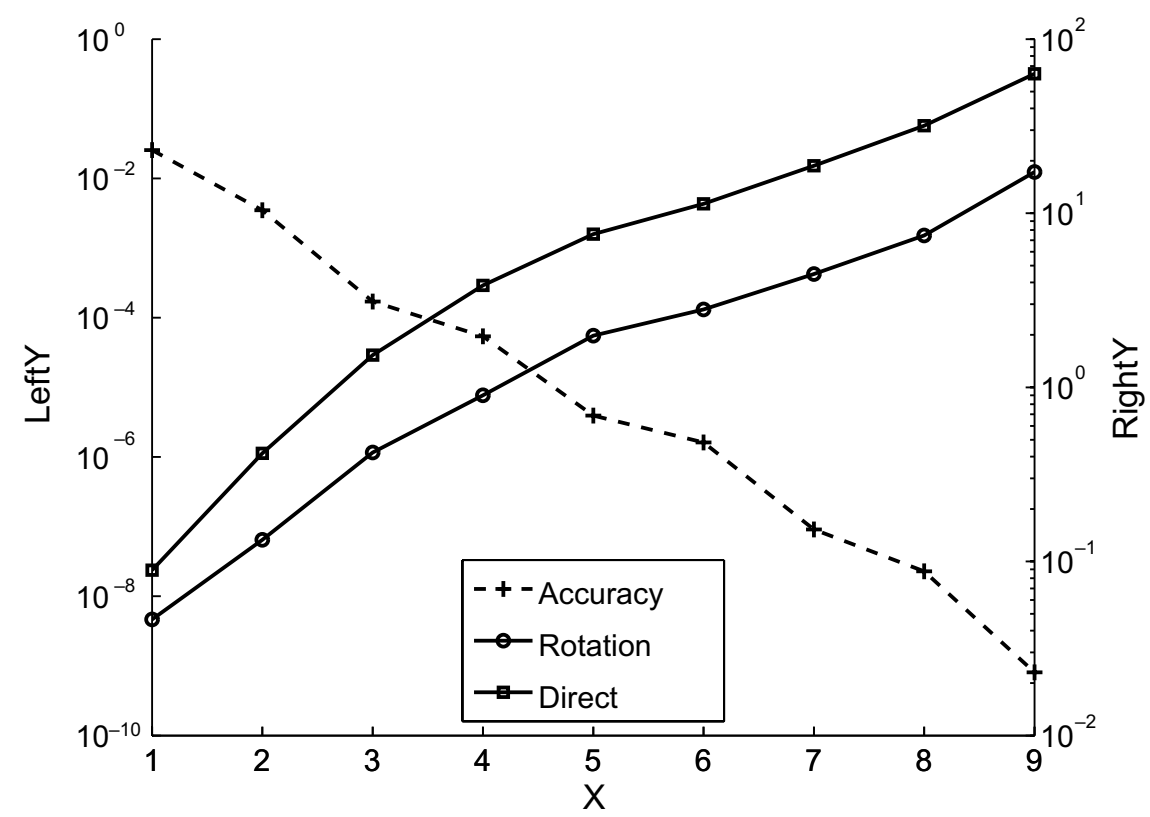

Fig. 6. The accuracy and time required for 1 iteration as a function of the multipole order $L$. The scattering geometry is shown in 5 . 
were calculated, so that the results would be more representative for larger scattering problems. The stable plane wave method used one level with translations. Fig. 6 shows a logarithmic plot of the iteration time and accuracy as a function of the multipole order for both the direct aggregation method and the method from Section 4.2. From 6 it is visible that the acceleration in this problem saturates at approximately a factor four. This is caused by the fact that the other operations (near interactions, translation) also add computational cost. Since these operations are not accelerated, this has a negative effect on the acceleration.

\section{Conclusion}

A novel method has been proposed that accelerates the multipole-to-plane-wave and plane-wave-to-multipole operations in the stable plane wave method. The fact that the Wigner D-matrices become diagonal for rotations around the $z$-axis has been exploited to obtain a acceleration of a factor six. Apart from some overhead which becomes negligible for not-too-small multipole orders, this reduces the computational cost of the six (dis)aggregations of the stable plane wave method to the cost of only one. The method has also been extended to the vectorial case and to the case with $N$ axes. For the vectorial case, two possible methods have been proposed. The first is very similar to the scalar case, the second uses the Beltrami decomposition of the electromagnetic field. This property permits splitting the vectorial MLFMA into two completely independent MLFMAs, including the (dis)aggregation from multipoles. As a consequence, some of the overhead associated with the new method is eliminated and this results in a method which is faster than the direct approach for any multipole order. These results have been shown both theoretically and numerically. The Beltrami decomposition is also valid for any vectorial MLFMA, still yielding an acceleration of a factor two.

\section{Acknowledgements}

The work of I. Bogaert was supported by a doctoral grant from the Institute for the Promotion of Innovation through Science and Technology in Flanders (IWT-Vlaanderen). D. Pissoort acknowledges the Fund for Scientific Research - Flanders (Belgium) (FWO-Vlaanderen) for a postdoctoral fellowship. The authors also thank K. Cools for useful discussions.

\section{Appendix}

For the definition of the spherical harmonics we will follow the conventions from [13]:

$$
\begin{aligned}
& Y_{l, m}(\boldsymbol{k})=(-1)^{m}\left(\frac{k_{x}+j k_{y}}{k}\right)^{m} K_{l m} P_{l}^{m}\left(\frac{k_{z}}{k}\right), \\
& Y_{l,-m}(\boldsymbol{k})=\left(\frac{k_{x}-\mathrm{j} k_{y}}{k}\right)^{m} K_{l m} P_{l}^{m}\left(\frac{k_{z}}{k}\right),
\end{aligned}
$$

with $m \geqslant 0, k=\sqrt{\boldsymbol{k} \cdot \boldsymbol{k}}$,

$$
K_{l m}=\sqrt{\frac{2 l+1}{4 \pi} \frac{(l-m) !}{(l+m) !}}
$$

and

$$
P_{l}^{m}(t)=\frac{\mathrm{d}^{m}}{\mathrm{~d} t^{m}} P_{l}(t)
$$

Here $P_{l}(t)$ is a Legendre polynomial. Some of the properties of spherical harmonics are:

$$
\begin{aligned}
& (-1)^{m} Y_{l,-m}(\boldsymbol{k})=\left[Y_{l, m}\left(\boldsymbol{k}^{*}\right)\right]^{*} \equiv Y_{l, m}^{*}\left(\boldsymbol{k}^{*}\right), \\
& Y_{l, m}(-\boldsymbol{k})=(-1)^{l} Y_{l, m}(\boldsymbol{k}) .
\end{aligned}
$$


The scalar multipoles are given by

$$
\Upsilon_{l, m}^{f}(\gamma \boldsymbol{r})=f(\gamma r) Y_{l, m}(\boldsymbol{r}) .
$$

Here $f(\cdot)$ denotes a spherical Hankel function $h_{l}^{(2)}(\cdot)$ or a spherical Bessel function $j_{l}(\cdot)$.

While the scalar multipoles are appropriate to describe scalar fields, vector multipoles are the most efficient way to describe vector fields arising in the general electromagnetic case. First the vector spherical harmonics will be introduced, followed by the vector multipoles. Since the electromagnetic field is divergence-free in sourcefree regions, only two sets of vector spherical harmonics are needed:

$$
\begin{aligned}
\boldsymbol{X}_{l, m}(\boldsymbol{k}) & =\frac{\hat{\boldsymbol{L}} Y_{l, m}(\boldsymbol{k})}{\sqrt{l(l+1)}}, \\
\boldsymbol{\Psi}_{l, m}(\boldsymbol{k}) & =\frac{\boldsymbol{k}}{k} \times \boldsymbol{X}_{l, m}(\boldsymbol{k}),
\end{aligned}
$$

with

$$
\hat{\boldsymbol{L}}=-\mathrm{j} \boldsymbol{r} \times \boldsymbol{\nabla}=\mathrm{j}\left[\boldsymbol{e}_{\theta} \frac{1}{\sin \theta} \partial_{\phi}-\boldsymbol{e}_{\phi} \partial_{\theta}\right] .
$$

Obviously, $\boldsymbol{X}_{l, m}(\boldsymbol{k})$ and $\boldsymbol{\Psi}_{l, m}(\boldsymbol{k})$ do not have radial components and their properties are easily deduced from the properties of spherical harmonics:

$$
\begin{aligned}
& (-1)^{m+1} \boldsymbol{X}_{l,-m}(\boldsymbol{k})=\boldsymbol{X}_{l, m}^{*}\left(\boldsymbol{k}^{*}\right), \\
& (-1)^{m+1} \boldsymbol{\Psi}_{l,-m}(\boldsymbol{k})=\boldsymbol{\Psi}_{l, m}^{*}\left(\boldsymbol{k}^{*}\right), \\
& \boldsymbol{X}_{l, m}(-\boldsymbol{k})=(-1)^{l} \boldsymbol{X}_{l, m}(\boldsymbol{k}), \\
& \boldsymbol{\Psi}_{l, m}(-\boldsymbol{k})=(-1)^{l+1} \boldsymbol{\Psi}_{l, m}(\boldsymbol{k}), \\
& \boldsymbol{X}_{l, m}(\boldsymbol{k}) \cdot \boldsymbol{X}_{l^{\prime}, m^{\prime}}(\boldsymbol{k})=\boldsymbol{\Psi}_{l, m}(\boldsymbol{k}) \cdot \boldsymbol{\Psi}_{l^{\prime}, m^{\prime}}(\boldsymbol{k}), \\
& \boldsymbol{X}_{l, m}(\boldsymbol{k}) \cdot \boldsymbol{\Psi}_{l^{\prime}, m^{\prime}}(\boldsymbol{k})=-\boldsymbol{\Psi}_{l, m}(\boldsymbol{k}) \cdot \boldsymbol{X}_{l^{\prime}, m^{\prime}}(\boldsymbol{k}) .
\end{aligned}
$$

The vector multipoles are defined as in Wittmann [13]:

$$
\begin{aligned}
& \boldsymbol{M}_{l, m}^{f}(\gamma \boldsymbol{r})=f(\gamma r) \boldsymbol{X}_{l, m}(\boldsymbol{r}) \\
& \boldsymbol{N}_{l, m}^{f}(\gamma \boldsymbol{r})=\frac{1}{\gamma} \boldsymbol{\nabla} \times \boldsymbol{M}_{l, m}^{f}(\gamma \boldsymbol{r})
\end{aligned}
$$

with $f$ again a spherical Bessel or Hankel function.

Rotations will be defined by the $3 \times 3$ matrix $\mathbf{R}(\alpha, \boldsymbol{a})$ which does the active rotation of a 3 -vector around an axis $\boldsymbol{a}$ (with $\boldsymbol{a} \cdot \boldsymbol{a}=1$ ) over the angle $\alpha$. An alternative representation is the Wigner D-matrix, which will be defined in this paper as:

$$
Y_{l, m_{1}}(\mathbf{R} \boldsymbol{r})=\sum_{m_{2}=-l}^{l} D_{m_{1}, m_{2}}^{l}(\mathbf{R}) Y_{l, m_{2}}(\boldsymbol{r})
$$

These Wigner D-matrices can be calculated very efficiently with a recursive algorithm described in [19]. A useful property of the Wigner D-matrices is that they become diagonal in the case of a rotation around the $z$-axis:

$$
D_{m, m^{\prime}}^{l}\left(\mathbf{R}\left(\theta, \boldsymbol{u}_{z}\right)\right)=\mathrm{e}^{\mathrm{i} m \theta} \delta_{m, m^{\prime}}
$$

The vector spherical harmonics transform under a rotation as follows:

$$
\begin{aligned}
& \overline{\overline{\boldsymbol{R}}}^{-1} \boldsymbol{X}_{l, m_{1}}(\overline{\overline{\boldsymbol{R}}} \boldsymbol{r})=\sum_{m_{2}=-l}^{l} D_{m_{1}, m_{2}}^{l}(\overline{\overline{\boldsymbol{R}}}) \boldsymbol{X}_{l, m_{2}}(\boldsymbol{r}) \\
& \overline{\boldsymbol{R}}^{-1} \boldsymbol{\Psi}_{l, m_{1}}(\overline{\overline{\boldsymbol{R}}} \boldsymbol{r})=\sum_{m_{2}=-l}^{l} D_{m_{1}, m_{2}}^{l}(\overline{\overline{\boldsymbol{R}}}) \boldsymbol{\Psi}_{l, m_{2}}(\boldsymbol{r})
\end{aligned}
$$




\section{References}

[1] V. Rokhlin, Rapid solution of integral equations of classical potential theory, J. Comput. Phys. 60 (1985).

[2] L. Greengard, The Rapid Evaluation of Potential Fields in Particle Systems, The MIT Press, 1987.

[3] R. Coifman, V. Rokhlin, S. Wandzura, The fast multipole method for the wave equation: a pedestrian prescription, IEEE Antennas Propag. Mag. 35 (1993) 7-12.

[4] L. Greengard, J. Huang, V. Rokhlin, S. Wandzura, Accelerating fast multipole methods for the helmholtz equation at low frequencies, Comput. Sci. Eng. 5 (3) (1998) 32-38.

[5] W. Chew, J. Jin, E. Michielssen, J. Song, Fast and Efficient Algorithms in Computational Electromagnetics, Artech House, 2001.

[6] E. Darve, P. Havé, A fast multipole method for Maxwell equations stable at all frequencies, Phil. Trans. R. Soc. Lond. A 362 (1816) (2004) 603-628.

[7] I. Bogaert, D. Pissoort, F. Olyslager, A normalized plane wave method for 2d Helmholtz problems, Microwave Opt. Technol. Lett. 48 (2) (2006) 237-243.

[8] E. Darve, P. Havé, Efficient fast multipole method for low-frequency scattering, J. Comput. Phys. 197 (1) (2004) $341-363$.

[9] H. Cheng, W.Y. Crutchfield, Z. Gimbutas, L.F. Greengard, J.F. Ethridge, J. Huang, V. Rokhlin, N. Yarvin, J. Zhao, A wideband fast multipole method for the helmholtz equation in three dimensions, J. Comput. Phys. (216) (2006) 300-325.

[10] L. Jiang, W. Chew, A mixed-form fast multipole algorithm, IEEE Trans. Antennas Propag. 53 (12) (2005) $4145-4156$.

[11] L. Jiang, W. Chew, Low-frequency fast inhomogeneous plane-wave algorithm (LF-FIPWA), Micro. Opt. Tech. Lett. 40 (2) (2004) $117-122$.

[12] T. Eibert, A diagonalized multilevel fast multipole method with spherical harmonics expansion of the $k$-space integrals, IEEE Trans. Antennas Propag. 53 (2) (2005) 814-817.

[13] R. Wittmann, Spherical wave operators and the translation formulas, IEEE Trans. Antennas Propag. 36 (8) (1988) $1078-1087$.

[14] W. Chew, Y. Wang, Efficient ways to compute the vector addition theorem, J. Electromagn. Waves Appl. 7 (5) (1993) $651-665$.

[15] A. Lakhtakia, Beltrami Fields in Chiral Media, World Scientific Publishing Co. Pte. Ltd., P.O. Box 128, Farrer Road, Singapore 9128, 1994.

[16] A. Lakhtakia, V. Varadan, V. Varadan, Time-Harmonic Electromagnetic Fields in Chiral Media, World Scientific Publishing Co. Pte. Ltd., P.O. Box 128, Farrer Road, Singapore 9128, 1994.

[17] I. Bogaert, F. Olyslager, Full-Wave Analysis of a finite piece of metamaterial, in: International Student Seminar on Microwave Applications of Novel Physical Phenomena, Rovaniemi, Finland, 2006.

[18] M.I. Mishchenko, J.W. Hovenier, L.D. Travis, Light Scattering by Nonspherical Particles: Theory, Measurements, and Applications, Academic Press, San Diego, 2000.

[19] C. Choi, J. Ivanic, M. Gordon, K. Ruedenberg, Rapid and stable determination of rotation matrices between spherical harmonics by direct recursion, J. Chem. Phys. 111 (19) (1999) 8825-8831. 